\title{
Axions and other (Pseudo) Goldstone Bosons
}

\section{Eduard Massó*}

Grup de Física Teòrica and IFAE

Universitat Autònoma de Barcelona

08193 Bellaterra, Barcelona, Spain

E-mail: masso@ifae.esi

ABSTRACT: I review the physics of axions and discuss some recent developments on pseudo-Goldstone bosons

\section{Introduction}

The QCD Lagrangian contains the so-called $\theta$-term

$$
\theta_{Q C D} \frac{\alpha_{s}}{8 \pi} G_{\mu \nu}^{a} \widetilde{G}^{a \mu \nu}+\ldots
$$

with $\widetilde{G}_{\mu \nu}^{a}=(1 / 2) \epsilon_{\mu \nu \rho \sigma} G^{a \rho \sigma}$, which is CP-violating. One may modify the value of $\theta$ by performing axial $U(1)_{A}$ rotations on the quarks. In turn, these rotations change the value of the phase of the determinant of the quark mass matrix $M$. In fact, there is a combination that is invariant under $U(1)_{A}$ rotations,

$$
\bar{\theta}=\theta_{Q C D}+\operatorname{Arg} \operatorname{Det} M
$$

A non-zero $\bar{\theta}$ would imply CP violation, most notably it would endow the neutron with an electric dipole moment

$$
d_{n} \sim \frac{e}{m_{n}} \bar{\theta} \frac{m_{u} m_{d}}{m_{u}+m_{d}} \frac{1}{\Lambda_{Q C D}}
$$

The experimental bound

$$
d_{n}<0.63 \times 10^{-25} e \mathrm{~cm}
$$

leads to

$$
\bar{\theta}<10^{-9}
$$

Why is $\bar{\theta}$ so small? We would have expected $\theta_{Q C D}$ and $\operatorname{Arg}$ Det $M$ not far from $\mathrm{O}(1)$, and we have no reason to expect such fine-tuned cancellation between the two terms $\theta_{Q C D}$

${ }^{*}$ Speaker. 
and $\operatorname{Arg}$ Det $M$, since they have totally unrelated origins. This is the so-called strong CP-problem.

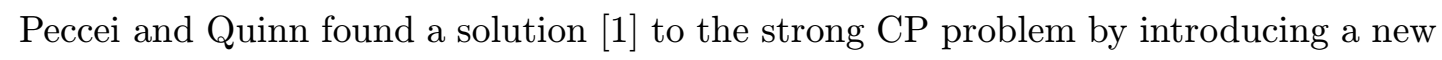
global chiral symmetry $U(1)_{P Q}$ and using the freedom to rotate $\bar{\theta}$ away. The spontaneous symmetry breaking of $U(1)_{P Q}$ at energy $\sim f_{a}$ generates a Goldstone boson: the axion, $a \sim f_{a} \bar{\theta}[\overline{\overline{2}}]$. We should keep in mind however that the PQ solution to the strong CP-

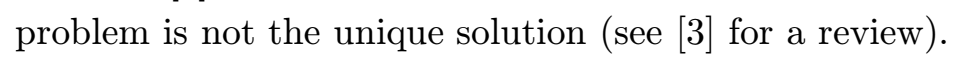

The axion couples derivatively to matter

$$
\mathcal{L}_{a \Psi \Psi}=\sum_{i} c_{i} \frac{1}{2 f_{a}}\left(\bar{\Psi}_{i} \gamma^{\mu} \gamma_{5} \Psi_{i}\right)\left(\partial_{\mu} a\right)
$$

Here $i=e, p, n$, etc, and $c_{i}=O(1)$ are model dependent parameters. The axion has also a non-derivative coupling to two gluons,

$$
\mathcal{L}_{a g g}=\frac{1}{f_{a}} \frac{\alpha_{s}}{8 \pi} G \cdot \widetilde{G} a
$$

At low $\left(\Lambda_{Q C D}\right)$ energies, the $g g a$ term gives rise to the potential $V(\bar{\theta})$ that makes $\bar{\theta} \rightarrow 0$ and also generates a mass for the axion

$$
m_{a}=\frac{f_{\pi} m_{\pi}}{f_{a}} \frac{\sqrt{m_{u} m_{d}}}{m_{u}+m_{d}}=0.6 \mathrm{eV} \frac{10^{7} \mathrm{GeV}}{f_{a}}
$$

Due to these properties the axion is not exactly a Goldstone boson (which is exactly massless and has only derivative couplings).

The axion has also an effective coupling to two photons:

$$
\mathcal{L}_{a \gamma \gamma}=c_{\gamma} \frac{\alpha}{\pi f_{a}} F \cdot \tilde{F} a=-g_{a \gamma \gamma} \vec{E} \vec{B} a
$$

Such a coupling is important from the point of view of a possible detection.

Let us stress that all $c_{i}$ are mildly model dependent except for the electron $c_{e}$ parameter. Indeed, there are models with $c_{e}=0$, i.e., the axion is not coupled to $e$ at tree level (KSVZ type or "hadronic axion") [忽]. However, most models have $c_{e} \neq 0$, for example in GUTembedded models like the DFSZ type [- Let us check, as a way of example, that the $a \gamma \gamma$ coupling is not wildly model dependent. For the DFSZ-type axion we have $c_{\gamma}=0.36$ and for the KSVZ-type we have $c_{\gamma}=-0.97$.

\section{Status of the axion}

The scale $f_{a}$ has to be a very high energy scale. This is an empirical fact that we know when using the constraints coming from particle physics experiments and astrophysical observations. Since $f_{a}$ and $m_{a}$ are related (1. $\left.1 . \mathbf{g}^{\prime}\right)$, this imply that the axion has to be very light. We also see, from the axion couplings showed in the last section, that it must be a very weakly interacting particle. 
Particle physics experiments, namely, decays involving axions or beam dump experiments lead to

$$
f_{a}>10^{4} \mathrm{GeV}
$$

or, equivalently,

$$
m_{a}<1 \mathrm{keV}
$$

This excludes that $f_{a}$ could be on the order of the Fermi scale, which was the original suggestion of Peccei and Quinn [i]

Astrophysical limits are obtained when considering that a "too" efficient energy drain due to axion emission would be inconsistent with observation. Stringent limits come from horizontal branch stars in globular clusters. The main production is from the Primakov process $\gamma \gamma^{*} \rightarrow a$ where $\gamma^{*}$ corresponds to the electromagnetic field induced by protons and

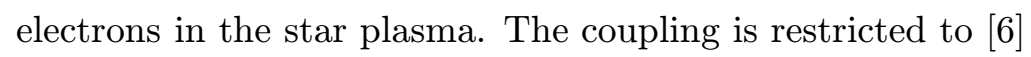

$$
g_{a \gamma \gamma}<0.6 \times 10^{-10} \mathrm{GeV} \Rightarrow f_{a}>10^{7} \mathrm{GeV}
$$

In terms of axion mass, the interval

$$
0.4 \mathrm{eV}<m_{a}<200 \mathrm{keV}
$$

is ruled out (for $m_{a}>200 \mathrm{keV}$ the axion is too heavy to be produced).

The most restrictive astrophysical limits on the axion parameters come from the analysis of neutrinos from the supernova SN 1987A. In the supernova core, the main production is axion bremsstrahlung in nucleon-nucleon processes, $N N \rightarrow N N a$. The observed duration of the $\nu$ signal at Earth detectors constrains the coupling of the axion to nucleons. The range

$$
3 \times 10^{-10}<g_{\text {ann }} \equiv c_{n} \frac{m_{n}}{f_{a}}<3 \times 10^{-7}
$$

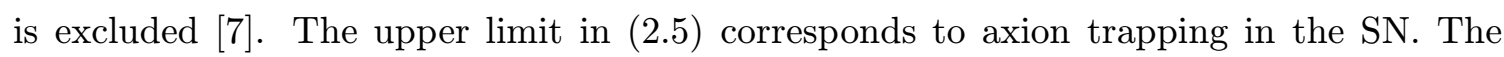
lower limit in $(2.5)$ is equivalent to $f_{a}>6 \times 10^{8} \mathrm{GeV}$. In terms of the axion mass, the excluded range corresponding to $(\overline{2} . \overline{2} \cdot \overline{1})$ is

$$
0.01 \mathrm{eV}<m_{a}<10 \mathrm{eV}
$$

Putting together all the information coming from laboratory and astrophysics we may conclude that the scale of the PQ breaking is bounded by

$$
f_{a}>6 \times 10^{8} \mathrm{GeV}
$$

A very exciting possibility is that, if the axion exists and its scale is not far from (2.7. it may be a substantial fraction of the dark matter of the universe. In the SSB of the PQ symmetry in the early universe, All vacuum expectation values $\langle a\rangle$ are equally likely, but naturally we expect $\langle a\rangle$ of the order of the PQ scale, or in other words an initial angle: $\bar{\theta}_{1} \sim<a>/ f_{a} \sim 1$. At the QCD scale, the mass of the axion turns on, and $\bar{\theta} \rightarrow 0$.

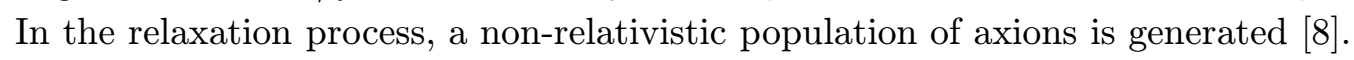


A careful estimate of the this energy density gives [igi]

$$
\Omega h^{2} \simeq 2 \times 10^{ \pm 0.4} F\left(\bar{\theta}_{1}\right) \bar{\theta}_{1}^{2}\left(\frac{10^{-6} \mathrm{eV}}{m_{a}}\right)^{1.18}
$$

where as usual, $\Omega$ is the density normalized to the critical density, and $h$ is the Hubble parameter today in units of $100 \mathrm{~km} / \mathrm{s} / \mathrm{Mpc} . F$ takes into account an-harmonic effects.

We see that a potentially interesting range for cosmology is

$$
\Omega h^{2} \sim 1-0.1 \Rightarrow m_{a} \sim 10^{-3}-10^{-6} \mathrm{eV}
$$

since then the axion could be part of the cold dark matter of the universe.

If we have as initial condition $F\left(\bar{\theta}_{1}\right) \bar{\theta}_{1}^{2} \sim 1$, we get a lower bound on the axion mass

$$
10^{-6} \mathrm{eV}<m_{a}
$$

However, for smaller values of the initial $\bar{\theta}_{1}$, one gets a looser bound. Apart from the value of $\bar{\theta}_{1}$, there are other cosmological uncertainties.

Another axion source is the string-produced axions. Unless inflation occurs at $T<f_{a}$, axion strings survive and decay into axions. For many years, there has been a debate on the importance of the string mechanism, and the question is not yet settled. On the one hand, some authors find that $\Omega_{\text {string }} \sim \Omega_{\text {misalign }}$ but some other authors find $\Omega_{\text {string }} \sim 10 \Omega_{\text {misalign }}$. Also, axion domain walls constitute another potential axion source. For a review and

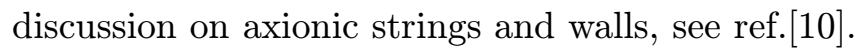

\section{Axion experiments}

The effort to detect axions is based in the following phenomena [i] $\left.\overline{1}_{1}^{1}\right]$. The interaction term (1.9.9) leads to axion-photon mixing in an external magnetic field. In addition, the probability of the $a-\gamma$ transition is enhanced when the $a-\gamma$ conversion in the magnetic field is coherent.

For example, in the presence of a flux of galactic halo axions, we expect conversion of axions into photons in a cavity with a strong magnetic field (haloscope) [ [i $\left.{ }^{1} i_{1}^{1}\right]$. When the (tunable) frequency of a cavity mode equals the axion mass,

$$
h \nu=E \simeq m_{a}\left(1+\beta^{2} / 2\right) \quad \beta \sim 10^{-3}
$$

there is a resonant conversion into $\mu$-wave photons $(1 \mathrm{GHz}=4 \mu \mathrm{eV})$. Axions are supposed to be virialized in the halo with $\beta \sim 10^{-3}$, so that there should be a very small dispersion.

Earlier experiments [1] $[1]$ put some limits, and presently there is already a secondgeneration experiment running, the US large scale experiment [i] $\left.{ }_{1} \overline{1}\right]$, sensitive in the range

$$
2.9 \mu \mathrm{eV}<m_{a}<3.3 \mu \mathrm{eV}
$$

This experiment has already excluded the possibility that KSVZ axions constitute the whole of the galactic dark matter density,

$$
\rho=7.5 \times 10^{-25} \mathrm{~g} \mathrm{~cm}^{-3}
$$


In the near future, they expect to reach $1 \mu \mathrm{eV}<m_{a}<10 \mu \mathrm{eV}$ [1] $\left.3_{1}\right]$.

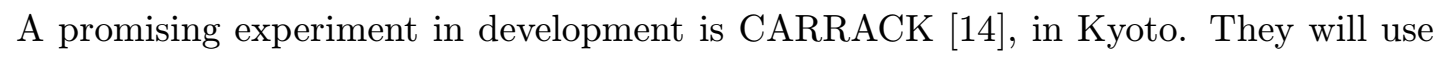
a Rydberg atoms' technique to detect $\mu$-wave photons.

Another alternative to detect axions makes use of the flux that the Sun would emit if axions exist, again making use of a strong magnetic field (helioscope) [1] $\left.\mathbf{1}_{-1}^{1}\right]$. The produced photons would have energies corresponding to the Sun interior, $E \sim$ a few $\mathrm{keV}$ (X-rays).

In Tokyo there is an experiment currently running, that has detected no signal, giving the limit [i] $[\overline{1} \overline{1}]$

$$
g_{a \gamma \gamma}<6 \times 10^{-10} \mathrm{GeV}^{-1}
$$

which is only valid for $m_{a}<0.03 \mathrm{eV}$, to preserve coherence. The Tokyo experiment has been improved recently by using gas to generate a plasmon mass $\omega_{\mathrm{pl}}$ and thus enhancing a possible signal for higher particle masses. They get [i] $\left.{ }_{1}^{i} \bar{i}\right]$

$$
g_{a \gamma \gamma}<6 \times 10^{-10} \mathrm{GeV}^{-1}
$$

now valid for $0.05 \mathrm{eV}<m_{a}<0.26 \mathrm{eV}$.

The CAST experiment at CERN [i] and is supposed to lead either to axion discovery or to very stringent limits.

An alternative way to convert axions from the Sun into photons is to use a crystal [i] $\left[\begin{array}{l}1 \\ \overline{8}\end{array}\right]$. Some experiments have lead to limits on the axion parameters [ī

Finally, we mention the axion laser searches, that make use of the effects on laser propagation that axions would produce. Stringent experimental limits are obtained [i $\left.\overline{2}_{0} \overline{0}\right]$.

\section{Axion-like particles}

As it is well known, a potential

$$
V=V_{\text {sym }}
$$

having an exact global symmetry, when spontaneously broken implies the appearance of (massless) Goldstone bosons. Example are family symmetries and lepton-number symmetry, that would give rise to familons and majorons.

However, global symmetries are expected to be broken by gravitation, since black holes do not conserve global charges. The total potential would read in this case

$$
V=V_{\mathrm{sym}}+V_{\text {non-sym }}
$$

with $V_{\text {non-sym }}$ suppressed by $M_{P}$ inverse powers.

In recent work we have been studying about the consequences of the spontaneous breaking of the symmetry in presence of (small) gravitationally-induced explicit breaking, particularly in phase transitions in the early universe.

As a simple case, take a global $U(1)$, with a scalar field $\Psi$

$$
V_{\mathrm{sym}}=\lambda\left[|\Psi|^{2}-v^{2}\right]^{2}
$$


and add a small breaking

$$
V_{\text {non-sym }}=\frac{g}{M_{P}^{n-2}}|\Psi|^{n} \Psi^{2}+\text { h.c. }
$$

with $n \geq 3$.

It is still convenient to parameterize

$$
\Psi=[v+\rho] \exp (i \theta / v)
$$

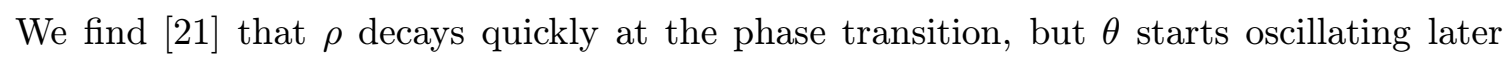
and gives rise to non-relativistic massive particles. For some range of parameters these pseudo-Goldstone bosons may constitute dark matter. In general, all these bosons couple to photons, so that they could give signatures in most of the experiments that look for axions .

\section{Conclusions}

The axion is a particle that is theoretically motivated, since is the consequence of the Peccei-Quinn solution to the strong CP problem. Axion physics is a very rich subject, particularly since it is possible that it constitutes part of the universal dark matter. Upcoming experiments may find the axion, or may exclude it. Other massive pseudo-Goldstone bosons have also interesting phenomenologies.

\section{Acknowledgments}

Work partially supported by the CICYT Research Project FPA2002-00648, by the EU network on Supersymmetry and the Early Universe HPRN-CT-2000-00152, and by the DURSI Research Project 2001SGR00188.

\section{References}

[1] R. D. Peccei and H. R. Quinn, Phys. Rev. Lett. 38 (1977) 1440.

R. D. Peccei and H. R. Quinn, Phys. Rev. D 16 (1977) 1791.

[2] S. Weinberg, Phys. Rev. Lett. 40 (1978) 223.

F. Wilczek, Phys. Rev. Lett. 40 (1978) 279.

[3] For a review, see H. Y. Cheng, Phys. Rept. 158 (1988) 1.

[4] J. E. Kim, Phys. Rev. Lett. 43 (1979) 103.

M. A. Shifman, A. I. Vainshtein and V. I. Zakharov, Nucl. Phys. B 166 (1980) 493.

[5] M. Dine, W. Fischler and M. Srednicki, Phys. Lett. B 104 (1981) 199.

A. R. Zhitnitsky, Sov. J. Nucl. Phys. 31 (1980) 260 [Yad. Fiz. 31 (1980) 497].

[6] G. G. Raffelt, "Stars As Laboratories For Fundamental Physics: The Astrophysics Of Neutrinos, Axions, And Other Weakly Interacting Particles," Chicago, USA: Univ. Pr. (1996) $664 p$. 
[7] J. R. Ellis and K. A. Olive, Phys. Lett. B 193 (1987) 525.

G. Raffelt and D. Seckel, Phys. Rev. Lett. 60 (1988) 1793.

M. S. Turner, Phys. Rev. Lett. 60 (1988) 1797.

[8] J. Preskill, M. B. Wise and F. Wilczek, Phys. Lett. B 120 (1983) 127.

L. F. Abbott and P. Sikivie, ibid. 133.

M. Dine and W. Fischler, ibid. 137.

M. S. Turner, Phys. Rev. D 33 (1986) 889.

[9] M. S. Turner, Phys. Rev. D 33 (1986) 889.

[10] P. Sikivie, Nucl. Phys. Proc. Suppl. 87 (2000) 41.

[11] P. Sikivie, Phys. Rev. Lett. 51 (1983) 1415 [Erratum-ibid. 52 (1984) 695].

[12] W. U. Wuensch et al., Phys. Rev. D 40 (1989) 3153.

C. Hagmann, P. Sikivie, N. S. Sullivan and D. B. Tanner, Phys. Rev. D 42 (1990) 1297.

[13] S. Asztalos et al., Phys. Rev. D 64 (2001) 092003.

[14] K. Yamamoto et al., arXiv:hep-ph/0101200.

[15] S. Moriyama, M. Minowa, T. Namba, Y. Inoue, Y. Takasu and A. Yamamoto, Phys. Lett. B 434 (1998) 147.

[16] Y. Inoue, T. Namba, S. Moriyama, M. Minowa, Y. Takasu, T. Horiuchi and A. Yamamoto, Phys. Lett. B 536 (2002) 18.

[17] Home page:

http://axnd02.cern.ch/CAST/

[18] E. A. Paschos and K. Zioutas, Phys. Lett. B 323 (1994) 367.

[19] F. T. Avignone et al. [SOLAX Collaboration], Phys. Rev. Lett. 81 (1998) 5068.

A. Morales et al. [COSME Collaboration], Astropart. Phys. 16 (2002) 325.

[20] Y. Semertzidis et al., Phys. Rev. Lett. 64 (1990) 2988. F. Brandi et al., Nucl. Instrum. Meth. A 461 (2001) 329.

G. Cantatore, talk at 4th International Workshop On The Identification Of Dark Matter (IDM2002), York, England.

[21] E. Masso, F. Rota and G. Zsembinszki, in preparation. 